\title{
Robotic microlaryngeal surgery: a new retractor that provides improved access to the glottis
}

\author{
Jennifer P. Rodney ${ }^{*}$ and Nilesh R. Vasan
}

\begin{abstract}
Robotic surgery has become the standard of care for many procedures outside of otolaryngology, and now is gaining momentum within our specialty. The robot has several advantages to human hands, including removal of tremor and better access to lesions due to increased degree of movement of the articulated instruments. The glottis has rarely been addressed using robotics because access was previously thought to be difficult. We present a case report using the modular oral retractor system to perform robotic microlaryngeal surgery.
\end{abstract}

\section{Background}

Robotic surgery has become more common for many surgical procedures. It eliminates human tremor and can be less invasive than open procedures, often resulting in decreased hospital stay and faster recovery (Dogan et al. 2001; Menon et al. 2003). Unfortunately, the larynx has remained a difficult anatomical area to address with robotic surgery, limited by the size and space requirements of current robotic instrumentation. Transoral robotic surgery (TORS) has steadily been gaining ground in otolaryngology, specifically for oropharyngeal and supraglottic resections for both benign and malignant neoplasms as well as for sleep-disordered breathing. The larynx has remained a difficult problem area in robotic surgery, however, limited by the size and space requirements of the robotic arms. A small number of studies have assessed the potential of RMLS, all using different methods of exposure. A recurring theme during these investigations is that multiple instruments have to be used to obtain access to the glottis and space for the robotic instruments is limited (Dogan et al. 2001; Hockstein et al. 2005a, b). Traditional glottic surgery is limited to direct laryngoscopes, which do not allow wide field access to glottic lesions. Visualization is limited to the

\footnotetext{
*Correspondence: jenrodney@gmail.com

Department of Otorhinolaryngology, The University of Oklahoma Health Sciences Center, PO Box 26901, Oklahoma, OK 73126-0901, USA
}

small view provided through the laryngoscope using a microscope. We have developed a device, called the modular oral retractor (MOR) system that is able to easily obtain a view of the glottis with the robot and maximize space in the glottic region to allow the robotic arms to function (Fig. 1). The device will enable RMLS to be less cumbersome and a more reasonable option when compared to traditional methods of glottic surgery.

\section{Case report}

A 56 year-old man with a long history of smoking initially presented with progressive hoarseness and dysphagia for 8 months. He underwent direct laryngoscopy and biopsy at an outside facility which showed squamous hyperplasia of the bilateral true vocal folds and diffuse, severe supraglottic hypertrophy. Nasopharyngoscopy performed in our clinic revealed redundant supraglottic mucosa that prolapsed into the glottic airway with associated plicae ventricularis, retroflexed epiglottis and prolapsed aryepiglottic folds. It was not possible to view the glottis due to the redundant mucosa. The remainder of the head and neck exam was unremarkable. The patient was deemed a good candidate for robotic-assisted surgery using the MOR system. Informed consent was obtained for examination of the larynx and removal of the obstructing mucosa with a $\mathrm{CO}_{2}$ laser. He was consented for use of the MOR system under an IRB-approved protocol.

\section{Springer}




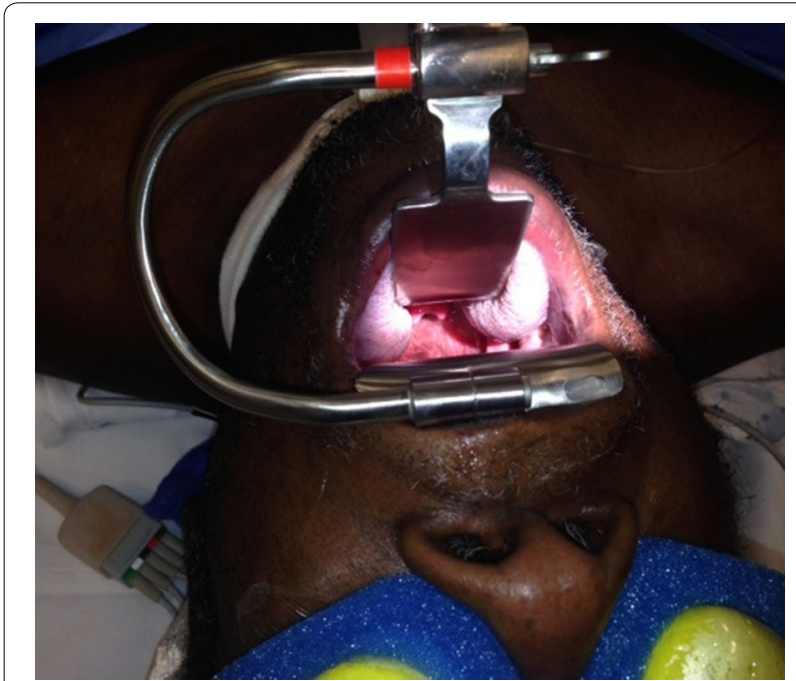

Fig. 1 The MOR system positioned for robotic microlaryngeal surgery

\section{Procedure}

After general anesthesia was induced and a shoulder roll was inserted, the MOR was inserted into the oral cavity using an appropriate curved blade and was suspended using the MOR suspension block. The retractor gave excellent access to the larynx (Fig. 2). The tongue blade used was a curved type that inserted into the vallecular space. There are multiple blade types available with the MOR system that takes into account the differences in anatomy between patients. When initially inserting the retractor using a headlight, the curve of the tongue blade provided elevation of the epiglottis that exposed the arytenoid mucosa. In some patients, more of the glottis larynx may be seen and these patients are easier candidates for robotic surgery. Following docking of the robot, the entire glottis including the anterior vocal folds could be visualized with the $30^{\circ}$ upward directed scope, but more

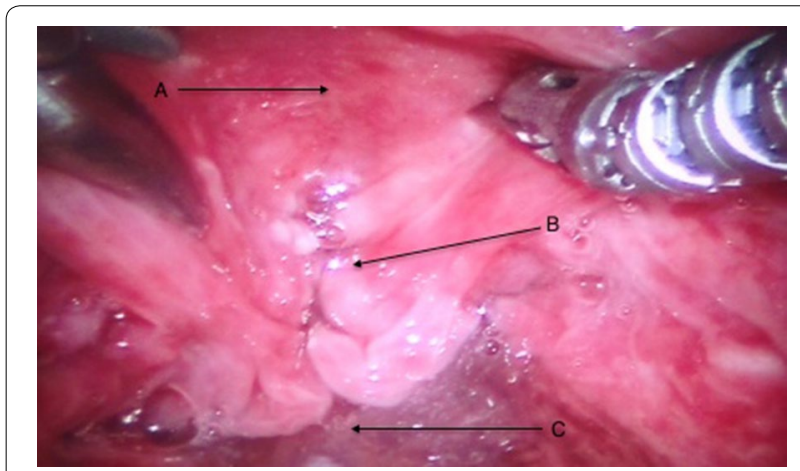

Fig. 2 Intraoperative photo; $A$ laryngeal surface of the epiglottis; $B$ redundant false ventricular tissue overlying the vocal folds; $C$ postcricoid area importantly, accessed by the robotic arms (Fig. 3). The improved exposure eliminated the need for a retraction tongue suture. The robot is docked with a Maryland dissector to the left and a needle driver on the right that allows mobilization and use of the OmniGuide $\mathrm{CO}_{2}$ laser. Setup using the retractor was relatively simple. The redundant mucosa on the superior aspect of the arytenoid cartilage on the left was excised completely using the laser at $15 \mathrm{~W}$. Unlike access obtained during microlaryngoscopy with a superior to inferior orientation within a narrow scope, tissue was able to be excised using the laser in a side-to-side manner, which is impossible with microlaryngoscopy. The MOR system therefore allows multiple surgical orientation options at the target tissue level. Hemostasis was achieved with the laser and suction cautery. A similar procedure was performed on the left side. The inter-arytenoid area was not treated to avoid contracture. The redundant mucosa could easily be mobilized with the Maryland forceps (Fig. 4). Because he patient had generalized edema, we elected to ablate the false cord on the left side, which was prominent. There were no complications during the procedure. The patient was monitored closely and had undergone multiple debridements of supraglottic tissue, including using a laryngoscope with dysplastic biopsies negative for malignancy. Six months postoperatively, a biopsy was positive for squamous cell carcinoma and he underwent total laryngectomy.

\section{Discussion}

Few in vivo studies have been reported to investigate the efficacy of RMLS. Two studies used the Dingman mouthgag and a $30^{\circ}$ scope to attain a working view of the larynx in a mannequin and cadaver, respectively (Hockstein et al. 2005a, b). Lalich et al. invented a retractor and conducted

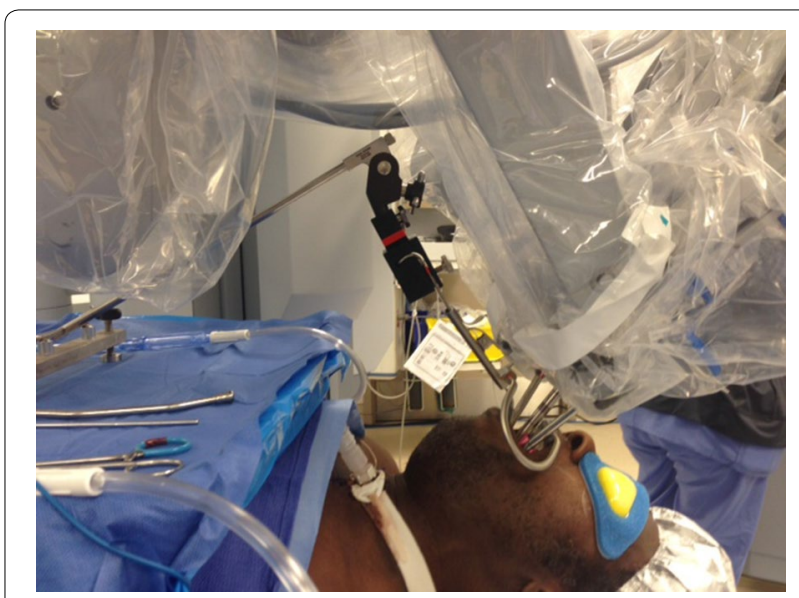

Fig. 3 The robot and the MOR system in position. $225 \times 169 \mathrm{~mm}$ $(72 \times 72 \mathrm{DPI})$ 


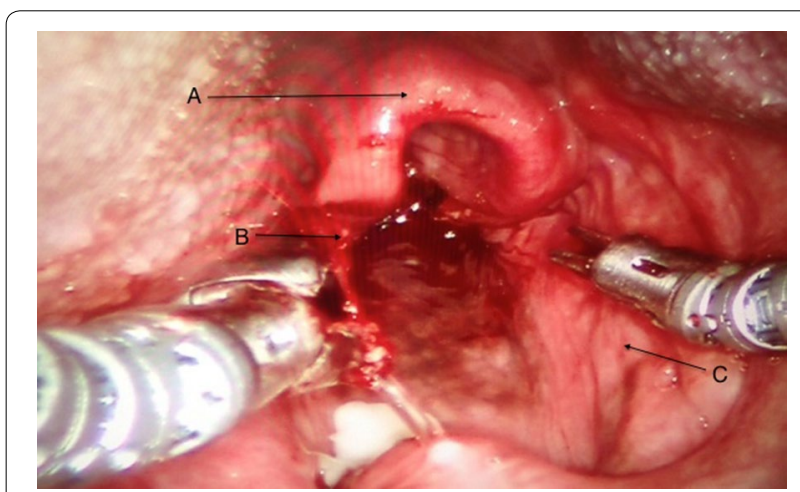

Fig. 4 Intraoperative photo demonstrating the robotic arms and exposure attained by the MOR system. $A$ epiglottis; $B$ aryepiglottic fold; C pyriformsinus. $215 \times 131 \mathrm{~mm}(72 \times 72)$

a study in cadavers with adequate access reported, but the retractor has not been trialed in an in vivo human model to date (Lalich et al. 2014). One study required the use of anterior tongue retraction with a 2.0 silk suture, malleable blade to retract the tongue base, and a Lindholm scope to retract the epiglottis. The robotic arms were inserted on either side of the Lindholm scope (Blanco et al. 2011). Resection of $\mathrm{T} 1$ glottic cancers have been successful in a small number of studies, but inadequate exposure was a recurrent theme (Park et al. 2009; Byrd and Duvvari 2013; Kayhan et al. 2012; Lallemant et al. 2013). An FK retractor was used in two studies, but the cumbersome nature of the retractor resulted in collision with the robotic arms and limited access to the anterior commissure in both studies. (Dogan et al. 2001; Blanco et al. 2011).

The senior author has used the Dingman, Crow Davis and FK retractor in the past and developed the MOR system as a simpler option with a wider range of applications. These previously described retractors are ideal to address the oropharynx particularly the palatine tonsil region but are limited in their exposure to other regions of the pharynx or larynx. The FK retractor is especially cumbersome in its use and its exposure of the larynx is very limited, especially in visualization of the anterior glottis. The MOR system was developed with two pivot points on the brace and a wide range of blade designs and can replicate the function of a Dingman or Crow Davis retractor. These features allow the user multiple set up options depending on the anatomical area being addressed. Our institution has not required use of the FK or any other retractor since the MOR became available. Unfortunately, direct comparisons between several retractors in a patient have not been performed due to the risk of unnecessary patient injury from insertion to removal of the retractor and significant operative time delay including use of the robot. A cadaver study in the future may have merit in this regard.
Our method showed successful robotic-assisted resection of glottic tissue using the MOR system. The MOR system eliminated the need for a rigid circular laryngoscope, which narrows the visual field, increases the distance of the working view to the surgical site and serves as an obstacle around which the robotic arms have to work. The MOR system did not require a stay suture to retract the tongue. Suturing the tongue can cause tongue edema, resulting in limited access and visualization of the surgical site as well as patient discomfort. The retractor has an axis of rotation at the base of the blade that optimizes elevation of the tongue and allows for retraction of the tongue down to the vallecula (Fig. 5). The MOR system includes 24 different blades each of which are customized to overcome commonly encountered anatomical challenges including a large tongue, large base of tongue, or an epiglottis that obscures view of the anterior glottis. The $360^{\circ}$ axis of rotation at the pivot of the base of the blade and the inferior portion of the mouth retractor allows

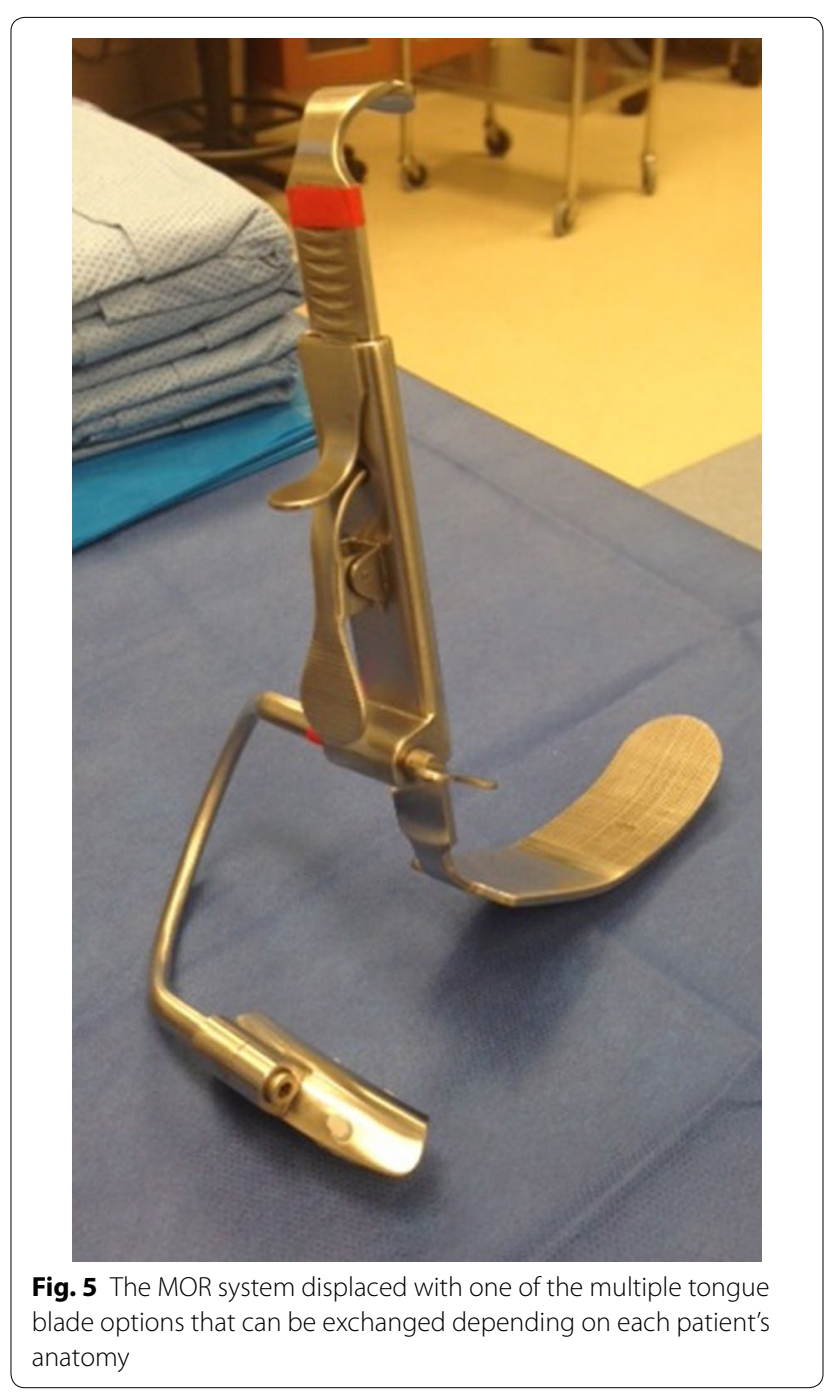


excellent exposure of the entire glottis which will likely eliminate the need for a tongue suture in most patients. The curvature of some of the available blades also serves to push the base of tongue anteriorly and allow adequate visualization of the glottis. The maxillary brace also has a $360^{\circ}$ axis of rotation that further augments the ability of the retractor to push the tongue and epiglottis forward, allowing visualization of the glottis (Fig. 6). The ability to rotate multiple parts of the retractor around an axis in order to maximize exposure is not possible with other retractors that have been proposed for use in robotic surgery. The robot eliminated tremor as a cause of potential human error and allowed for $360^{\circ}$ access to the lesions, which in our case was redundant supraglottic tissue that had prolapsed into the glottic airway.

Disadvantages of RMLS include limited instrumentation in which success varies depending on each patient's anatomy, limited tactile feedback requiring the surgeon to rely on visual cues, and limited robotic training programs. Oncologic outcomes using RMLS have not yet been studied or compared to traditional surgery (Byrd and Duvvari 2013). Limitations of this study include that the MOR system has only been evaluated in one case thus far, which limits its generalizability. It has not yet been tested across a wide variety of patient with different anatomy and body habitus. The blade on the retractor does not retract the epiglottis, which is sometimes necessary for exposure depending on the patient's anatomy. When using the MOR system, attention needs to be given to the size and width of the tongue blade so that the base of the vallecula is reached for optimal glottic exposure. In some cases, the epiglottis may need to be retracted for exposure. We have created multiple blade sizes to address this issue, including a blade similar to the superior aspect of a Lindholm blade that can connect with the MOR brace (Fig. 7). This modification of the Lindholm blade that is commonly used in microlaryngeal surgery gives the robotic surgeon similar access to the larynx. With the recent release of the new da Vinci Xi surgical robot, we believe the MOR system will be even simpler to use with wider application, as the robotic arm instruments are $5 \mathrm{~cm}$ longer and more slender.

\section{Conclusion}

RMLS using the MOR system shows promise to allow for easy and effective resection of glottic and supraglottic lesions. We propose that by using this retractor, RMLS may improve upon traditional techniques. Technological advancements such as the new da Vinci Xi robotic system may make the MOR system a better option to

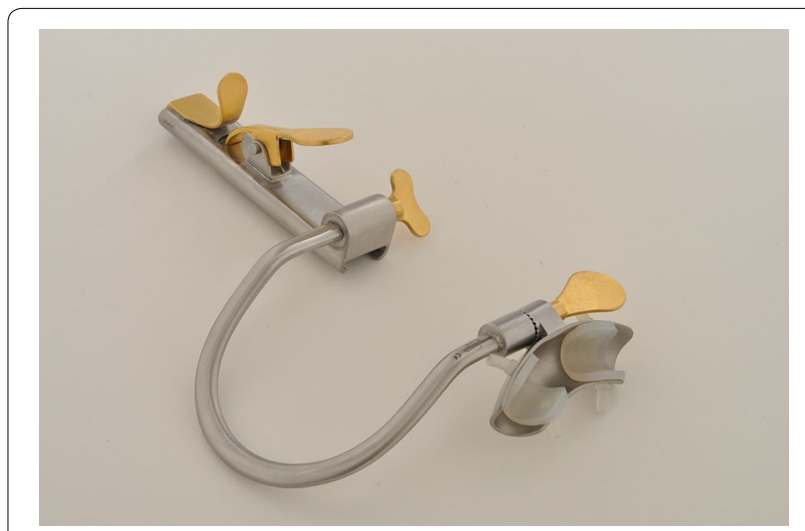

Fig. 6 Both the maxillary brace and pivot at the base of the blade have a $360^{\circ}$ axis of rotation which improves exposure of the glottis

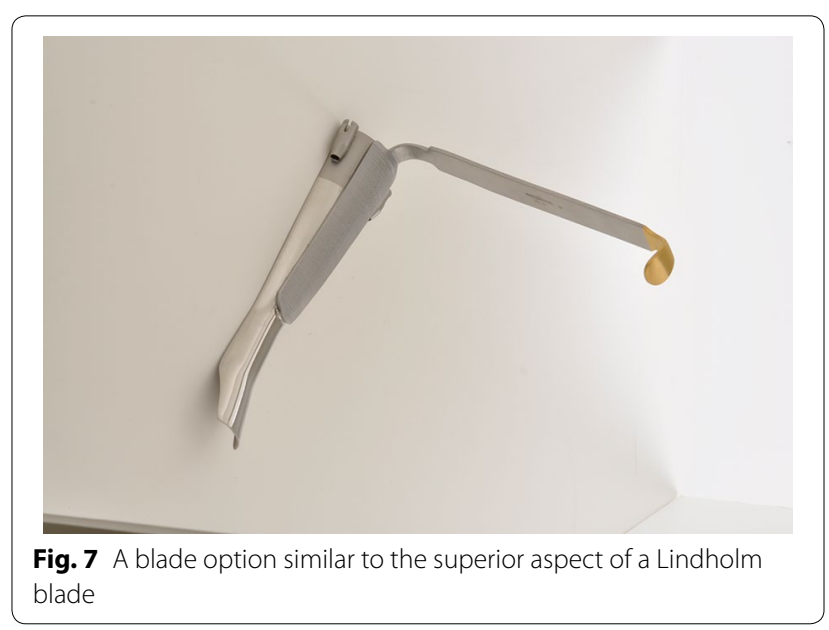

perform laryngopharyngeal surgery. Areas of future analysis include a case series of multiple lesions that have been removed via RMLS using the MOR system.

\section{Authors' contributions}

NV made substantial contributions to conception and design, analysis and interpretation of data. He was involved in revising the manuscript critically for important intellectual content and has given final approval of the version to be published. He agrees to be accountable for all aspects of the work in ensuring that questions related to the accuracy or integrity of any part of the work are appropriately investigated and resolved. JR made substantial contributions to conception and design, analysis and interpretation of data. She drafted the manuscript and has given final approval of the version to be published. She agrees to be accountable for all aspects of the work in ensuring that questions related to the accuracy or integrity of any part of the work are appropriately investigated and resolved. Both authors read and approved the final manuscript.

\section{Authors' Information}

Dr. Vasan is an Otolaryngologist—Head and Neck Surgeon in Oklahoma City at the University of Oklahoma Health Sciences Center (OUHSC). He was born and raised in New Zealand where he attended medical school and completed 
Residency. He is a Fellow of the Royal Australasian College of Surgeons. Dr. Vasan moved to Oklahoma City in 2002 where he completed a 2-year Head and Neck Fellowship at the OUHSC. He specializes in treating benign and malignant disease within the Head and Neck as well as General ENT disorders.

Dr. Vasan is the only physician in the state performing Head \&Neck Robotic surgery and is active in the field of medical device innovation. Dr. Vasan is the Residency Program Director for the Department of Otorhinolaryngology.

Dr. Jennifer Rodney is a third year resident in the University of Oklahoma Department of Otorhinolaryngology. She is originally from Saint Petersburg, Florida. She completed her undergraduate training at The University of South Florida, double majoring in voice performance and biomedical science, with a minor in Spanish Language. She completed medical school at The University of Florida and moved to Oklahoma for residency training. She is a professional classical singer and has performed throughout the Southeast and in Europe prior to medical school.

She plans to combine her two passions by completing a fellowship in laryngology after her residency training. Her scholarly interests include robotic microlaryngeal surgery, care of the professional voice, and both benign and malignant vocal fold disorders.

\section{Acknowledgements}

Marina Medical Inc. (Sunrise, FL) provided assistance in engineering and production of the MOR system.

Intuitive Surgical Incorporated provided grant funding for this investigation. Intuitive Surgical has awarded $\$ 5000$ for travel purposes for both authors to present research related to the MOR retractor. Intuitive Surgical Inc. did not have a role in the design, collection, analysis or interpretation of data.

This manuscript was presented at the Triological Society Combined Sections in Coronado Island, California, USA, 22-24 January 2015.

\section{Competing interests}

Nilesh Vasan, is the inventor of the retractor described in this case report with an International Patent application for the device filed in 2013 through the University of Oklahoma.

Jennifer Rodney does not have any conflicts of interest to disclose.

\section{Consent to publish}

Written consent to publish has been obtained from the participant to report individual patient data. The written consent is available for review by the Editor-in-Chief of this journal.

\section{Ethical approval}

All procedures performed in studies involving human participants were in accordance with the ethical standards of the institutional and/or national research committee and with the 1964 Helsinki declaration and its later amendments or comparable ethical standards. This article does not contain any studies with animals performed by any of the authors.

\section{Funding}

This case report did not require financial support. The authors have a clinical research grant from Intuitive Surgical, Inc. to continue to perform and present research using the MOR system.

\section{Informed consent}

Informed consent was obtained from all individual participants included in the study. Additional informed consent was obtained from the participant for whom identifying information is included in this article.

\section{Received: 17 August 2015 Accepted: 12 February 2016}

Published online: 27 February 2016

\section{References}

Blanco RG, Ha PK, Califano JA, Saunders JM (2011) Transoral robotic surgery of the vocal cord. J Laparoendosc Adv Surg Tech 21:157-159

Byrd JK, Duvvari U (2013) Current trends in robotic surgery for otolaryngology. Current Otorhinolaryngol Rep 1:153-157

Dogan S, Aybek T, Westphal K, Mierdi S, Moritz A, Wimmer-Greinecker G (2001) Computer-enhanced totally endoscopic sequential coronary artery bypass. Ann Thorac Surg 72:610-611

Hockstein NG, Nolan JP, O'Malley BW Jr, Woo YJ (2005a) Robot-assisted pharyngeal and laryngeal microsurgery: results of Robotic cadaver dissections. Laryngoscope 115:1003-1008

Hockstein NG, Nolan JP, O'Malley BW Jr, Woo YJ (2005b) Robotic microlaryngeal surgery: a technical feasibility study using the daVinci surgical robot and an airway mannequin. Laryngoscope 115:780-785

Kayhan FT, Kaya KH, Sayin I (2012) Transoral robotic cordectomy for early glottic carcinoma. Ann Otol Rhinol Laryngol 121:497-502

Lalich IJ, Olsen SM, Ekbom DC (2014) Robotic microlaryngeal surgery: feasability using a newly designed retractor and instrumentation. Laryngoscope 124:1624-1630

Lallemant B, Chambon G, Garrel R et al (2013) Transoral robotic surgery for the treatment of T1-T2 carcinoma of the larynx: preliminary study. Laryngoscope 123:2485-2490

Menon M, Shrivasta A, Sarle R et al (2003) Vattikuti Institute Prostatectomy: a single-team experience of 100 cases. J Endourol 17:785-790

Park YM, Lee WJ, Lee JG, Lee WS, Choi EC, Chung SM, Kim SH (2009) Transoral Robotic Surgery (TORS) in laryngeal and hypopharyngeal cancer. J Laparoendosc Adv Surg Tech 19:361-368

\section{Submit your manuscript to a SpringerOpen ${ }^{\odot}$ journal and benefit from:}

- Convenient online submission

- Rigorous peer review

- Immediate publication on acceptance

- Open access: articles freely available online

- High visibility within the field

- Retaining the copyright to your article

Submit your next manuscript at $>$ springeropen.com 